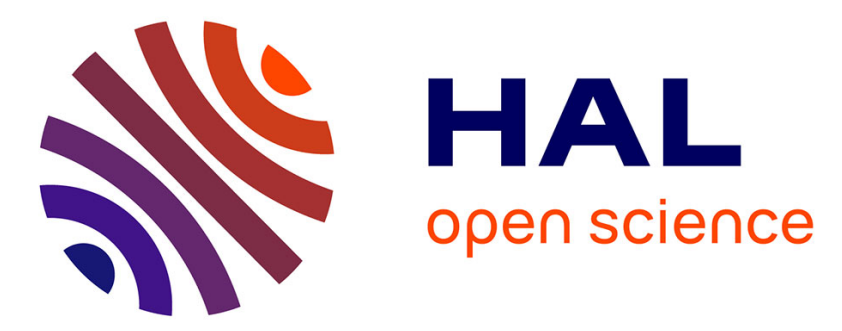

\title{
A three-dimensional semi-quantitative method to monitor the evolution of polycyclic aromatic hydrocarbons from vacuum gas oil feedstocks to lighter products
}

Carole Reymond, Agnès Le Masle, Cyril Colas, Nadège Charon

\section{To cite this version:}

Carole Reymond, Agnès Le Masle, Cyril Colas, Nadège Charon. A three-dimensional semi-quantitative method to monitor the evolution of polycyclic aromatic hydrocarbons from vacuum gas oil feedstocks to lighter products. Fuel, 2021, 296, pp.120175. 10.1016/j.fuel.2021.120175 . hal-03194183

\section{HAL Id: hal-03194183 \\ https://hal-ifp.archives-ouvertes.fr/hal-03194183}

Submitted on 9 Apr 2021

HAL is a multi-disciplinary open access archive for the deposit and dissemination of scientific research documents, whether they are published or not. The documents may come from teaching and research institutions in France or abroad, or from public or private research centers.
L'archive ouverte pluridisciplinaire HAL, est destinée au dépôt et à la diffusion de documents scientifiques de niveau recherche, publiés ou non, émanant des établissements d'enseignement et de recherche français ou étrangers, des laboratoires publics ou privés. 


\section{A three-dimensional semi-quantitative method to monitor the evolution of polycyclic} aromatic hydrocarbons from vacuum gas oil feedstocks to lighter products

Carole Reymond ${ }^{\mathrm{a}}$, Agnès Le Masle ${ }^{\mathrm{a},{ }^{*}}$, Cyril Colas $^{\mathrm{b}, \mathrm{c}}$, Nadège Charon $^{\mathrm{a}}$

a IFP Energies nouvelles, Rond-point de l'échangeur de Solaize, BP 3, 69360 Solaize, France

${ }^{\mathrm{b}}$ Institut de Chimie Organique et Analytique, Université d'Orléans, CNRS UMR 7311, Rue de Chartres, 45067 Orléans, France

${ }^{c}$ Centre de Biophysique Moléculaire, CNRS UPR 4301, Université d'Orléans, rue Charles Sadron, 45071 Orléans, France

*Corresponding author. E-mail address: agnes.le-masle@ifpen.fr (A. Le Masle)

\section{Abstract}

Formation and accumulation of polycyclic aromatic hydrocarbons (PAHs) are responsible of major topical issues related to the conversion of petroleum vacuum gas oils (VGOs) in transportation fuels through hydrocracking process. To achieve an efficient management of these hydrocarbons, a detailed semi-quantitative description of PAHs contained in VGOs was provided by a threedimensional off-line analytical approach based on centrifugal partition chromatography (CPC), supercritical fluid chromatography (SFC) and high-resolution mass spectrometry (HRMS). As a result, well-organised CPCXSFC chromatograms provided a relevant separation of PAHs according to both unsaturation level (DBE) and alkylation degree. This dedicated methodology allowed the semiquantification of PAHs having a DBE superior to 12. For the first time, PAHs were quantified in six VGOs allowing to highlight differences in composition depending on the origin of the samples. Then, the evolution of PAH concentration was monitored during hydrotreating and hydrocracking processes. For the hydrotreated/hydrocracked (HDT/HCK) products, their sulphur and nitrogen contents were low enough to make possible semi-quantification of PAHs directly by SFC/HRMS. An accumulation of PAHs was observed when a recycle stream from the unconverted oil to HDT reactor was applied. This work demonstrates that valuable analytical data could be obtained leading a step 
26 forward in the overall understanding of a process from native VGO feedstocks to the HDT/HCK

27 products.

28 Keywords

29 Semi-quantification ; polycyclic aromatic hydrocarbons ; vacuum gas oil ; hydrocracking ; highresolution mass spectrometry; two-dimensional liquid chromatography 
Today many topical issues have to be addressed in the energy field and one of them is to meet the increasing worldwide demand for transportation fuels while fulfilling narrow product specifications [1]. For this purpose, efforts are continuously made to improve performances of advanced conversion technologies such as hydrocracking (HCK) dedicated to the transformation of the heavy petroleum fractions to targeted light and middle distillates [2]. Vacuum gas oils (VGOs) are high boiling point petroleum cuts $\left(350-550^{\circ} \mathrm{C}\right)$ that are typically used as feedstocks in HCK units. They contain significant contents of sulphur and nitrogen compounds, known to act as poisons towards HCK catalysts, so a first hydrotreating step (HDT) is generally performed in order to remove these heteroelements in presence of an excess of hydrogen $[3,4]$. During this HDT step, olefins and many aromatic compounds may be saturated, leading to an increase of the number of hydrogen atoms/number of carbon atoms ratio $(\mathrm{H} / \mathrm{C})$. Once VGO has been hydrotreated, it is converted into lighter and valuable products by breaking the carbon-carbon bonds in a HCK reactor. There are several HCK configurations including single-stage or two-stage units, with or without recycle. In a once-through configuration, hydrotreated VGO corresponds to the unique hydrocarbons feedstock entering into the HCK reactor. In a recycle configuration, VGO goes through HDT and HCK reactors and then a part of the unconverted oil (UCO) resulting from the distillation of HCK products is used as a co-processing feedstock, mixed with the original VGO, at the inlet of either HDT or HCK reactor [5]. The objective of the recycle operation mode is to increase the conversion of VGO and thus to get more light products. Thanks to this advanced technology as well as to the improvement of the catalysts performances [6], the overall conversion yields exceed 95 wt\% [7]. However one great issue of hydrocracker operating with recycle of UCO is the formation and accumulation of polycyclic aromatic hydrocarbons (PAHs), and more specifically of heavy PAHs with more than eight aromatic rings. These heavy compounds are not initially present in native VGOs but come from unwanted reactions involving PAHs with 2-6 rings and their alkylated derivatives which are already contained in VGO feedstocks $[8,9]$. In a recycle configuration, PAHs are concentrated in UCO distillate and go back 
to the HCK reactor, which ultimately leads to coke formation, deactivation of catalysts and clogging issues in the recycle piping.

In order to optimise process conditions, analytical characterisation and kinetic modelling are required. In the literature, many modelling approaches for HCK of heavy oils have already been reported [10-17]. Most of them employ lumping techniques using macroscopic properties (e.g. density, boiling point, elemental analysis, etc.). However, the predictive power of this kind of modelling is limited and for example cannot take into account the PAH accumulation phenomenon. At the opposite, molecular models give reliable prediction by considering the molecular composition of feedstocks and products. One major impediment of this modelling approach is the availability of detailed chemical data. Although analytical devices become more and more powerful, petroleomics is still a challenge for chemical analysis [18]. Ultra high-resolution mass spectrometry such as Fouriertransform ion cyclotron resonance mass spectrometry (FT-ICR-MS) plays an important role in this research field by identifying several thousands of oil components and globally improving knowledge about HCK reactions [19-22]. Nevertheless, mass spectrometry alone cannot resolve the complexity of petroleum due to ionisation competition leading to qualitative data and the inability to differentiate isomeric species [23]. The use of ion mobility spectrometry-mass spectrometry (IMSMS) can be a solution to bring additional information on the structure and differentiate isomer species [24]. However, ionisation competition cannot be solved with IMS-MS. Using chromatographic separation is a relevant way to overcome this limitation; for instance, Panda et al. proposed a nonaqueous reversed phase chromatography (NARP) method to quantify PAHs in a recycle UCO stream [25]. Unfortunately this method is well-adapted to streams with low sulphur contents but is not applicable to VGO feedstocks. Supercritical fluid chromatography (SFC) is used to propose a quantification of aromatic families (non aromatic, monoaromatic and polynuclear aromatic) (ASTMD5186) or a quantification of olefin content (ASTM-D6550) in petroleum samples using a flame ionisation detector (D5186). High--temperature two-dimensional gas chromatography (HT-GCXGC) is also proposed to elute highly aromatic hydrocarbons like coronene but no molecular detailed 
quantification is described with FID [26-28]. The hyphenation with HRMS is necessary for mixture as complex as VGO.

In a previous work, we have proposed an off-line comprehensive three-dimensional methodology to quantify PAHs in VGO samples [29]. This method consists in using centrifugal partition chromatography (CPC) as a first dimension and SFC as a second dimension hyphenated to FT-ICR-MS. The ionisation of PAHs was performed thanks to an atmospheric pressure photoionisation (APPI) source operating in positive mode. Since PAHs have an ionisation energy smaller than $10 \mathrm{eV}$, their direct ionisation can be performed thanks to photons from the krypton lamp [30]. Moreover, several works showed that APPI in positive mode is one the most suitable and efficient atmospheric pressure ionisation source for PAHs ionisation [31-33]. The first dimension of the three-dimensional method, CPC, allows a separation according to the alkylation stage of the hydrocarbons whereas the second one, SFC, is based on their double bond equivalent (DBE). Thanks to this specific hyphenation, it has been demonstrated that no matrix effect occurs in the ionisation source and thus PAHs in VGOs could be quantified by mass spectrometry for the first time.

The objective of this study is to extend the quantification of PAHs to their alkylated derivatives and to apply the methodology to a diversified set of samples. Firstly, six VGOs coming from different process operating conditions were investigated and their PAH composition was described from a qualitative and semi-quantitative viewpoint. Secondly our analytical method was used to measure the evolution of PAH contents from a native VGO to hydrocracked UCO products in both once-through and recycle configurations.

\section{Experimental section}

\subsection{Samples}

Six VGO samples including four straight-run vacuum gas oils (SRVGO), one VGO coming from deep conversion (DCVGO) and one blend (SRVGO and DCVGO 50/50 v/v) were investigated in this study. 
Details on these samples are given in Table 1. Large ranges of nitrogen and sulphur contents are covered by the selected VGO, going from 2974 to $25792 \mathrm{mg} / \mathrm{kg}$ for sulphur contents and from 982 to $3510 \mathrm{mg} / \mathrm{kg}$ for nitrogen contents.

Process parameters during the sampling of recycle stream are detailed in Table 2.

\subsection{Chemicals}

All solvents were purchased from VWR (Fontenay-sous-Bois, France). Acetonitrile (ACN), heptane (HEPT), dichloromethane (DCM) and toluene were HPLC grade. Methanol (MeOH) used during SFC analyses was HPLC-MS grade. Anisole was $\geq 99 \%$ pure. Carbon dioxide SFC grade (99.97\%) (B50 cylinder under pressure) was purchased from Air Liquide (Paris, France).

Anthracene, pyrene, benz(a)anthracene, perylene, benzo(ghi)perylene and coronene were purchased from Sigma-Aldrich (Saint-Quentin-Fallavier, France). 1-methylpyrene, 4-methylpyrene, 4,5-dimethylpyrene, 2,7-dimethylpyrene, 1-ethylpyrene, 1-propylpyrene, 1-butylpyrene and 1-methylcoronene were purchased from BCP Instruments (Oullins, France).

\subsection{Methodology}

In a previous work, a three-dimensional method consisting in the off-line hyphenation of CPC and SFC/HRMS was developed [29]. The fractions collected from CPC separation were evaporated, then dissolved in $300 \mu \mathrm{L}$ of toluene and analysed by SFC/HRMS. For one sample, 40 base peak chromatograms (BPC) were extracted from the mass signal, corresponding to each CPC fraction. Thanks to a home-made software, 2D plot could be traced by combining the BPC. The complete methodology is presented on Figure 1.

\subsection{Centrifugal Partition Chromatography (CPC)}

CPC experiments were carried out with a SCPC100 associated to a Spot Prep II from Armen Instrument (Gilson Purification, USA). The system included an automatic sample injection with a $5 \mathrm{~mL}$ 
loop, a quaternary pump, a diode array detector (DAD) ranging from 200 to $400 \mathrm{~nm}$ and a fraction collector. A $131 \mathrm{~mL}$ column was used. Chromatographic data were managed using the Armen Glider CPC software. Experiments were conducted at room temperature.

Solvent system consisted in HEPT/DCM/ACN 45/10/45 (v/v/v). Biphasic system solvent was prepared in a separatory funnel. Descendant elution mode allowed to use the heptane-enriched phase as the stationary phase and the acetonitrile-enriched phase as the mobile phase. The stationary phase ratio was measured at $60 \%$. Samples were prepared as follows: $1 \mathrm{~g}$ of sample was diluted in $5 \mathrm{~mL}$ of stationary phase. After $10 \mathrm{~min}$ of equilibration with the mobile phase at $30 \mathrm{~mL} / \mathrm{min}$ and $500 \mathrm{rpm}$, elution took place during the first $30 \mathrm{~min}$ of the run $(2000 \mathrm{rpm}, 10 \mathrm{~mL} / \mathrm{min}$ of mobile phase), then the run ended with $10 \mathrm{~min}$ of extrusion (2000 rpm, $30 \mathrm{~mL} / \mathrm{min}$ of stationary phase). One fraction was collected each minute ( 40 fractions in total per run). These fractions were evaporated under vacuum to dryness using a Rocket Synergy evaporator (Biopharma Technologies, France) at $40^{\circ} \mathrm{C}$. The dry extracts were dissolved in $300 \mu \mathrm{L}$ of toluene for further analyses with SFC/HRMS.

\subsection{SFC/HRMS}

SFC experiments were carried out on an Acquity UPC (Waters, USA). The instrument control was performed by Empower 3 software (Waters). SFC method was optimised in a previous work [29]. Briefly, separations were achieved with a back pressure of $10.5 \mathrm{MPa}$ at $55^{\circ} \mathrm{C}$ on a Torus 2-PIC column ( $3 \times 100 \mathrm{~mm}$, particle size $1.7 \mu \mathrm{m}$, Waters). Methanol was used as co-solvent. The mobile phase flow rate was $1.1 \mathrm{~mL} / \mathrm{min}$. The method started with an isocratic step of 5 min with $100 \%$ of $\mathrm{CO}_{2}$, then the organic co-solvent ranged from 0 to $30 \%$ in $15 \mathrm{~min}$. The injection volume was $1 \mu \mathrm{L}$. The detection UV wavelengths ranged from 210 to $400 \mathrm{~nm}$. Finally, the flow was splited, one part going to the mass spectrometer and the other one to the waste via the automated back pressure regulator (ABPR).

The chromatographic system was hyphenated to a linear ion trap - Fourier-transform ion cyclotron resonance mass spectrometer (LTQ-FT-ICR Thermo Scientific, Germany) equipped with a 7 T magnet. Ionisation was carried out using APPI in positive mode. Anisole was employed as APPI dopant with a 
$100 \mu \mathrm{L} / \mathrm{min}$ flow rate at the outlet of the diode array detector (DAD). Ionisation and transfer

157 conditions were optimised with a design of experiments approach according to a previous work [29].

Sheath, auxiliary and sweep gas flow rates were set to 10, 34 and 0 A.U. respectively. APPI vaporiser and transfer capillary temperatures were equal to 348 and $200^{\circ} \mathrm{C}$ respectively. Transfer capillary and tube lens voltages were set to 48 and $100 \mathrm{~V}$ respectively. The mass range was set as $m / z$ 98-1000. In order to have at least 10 points to correctly describe each chromatographic peak, a resolving power of 12500 at $m / z 400$ and $2 \mu$ scans were used. External mass calibration was performed using Calmix from Thermo Fisher Scientific. The mass accuracy was better than $2 \mathrm{ppm}$.

\section{6. $\quad$ MS data processing}

Data were processed using MZmine 2.53 software [34]. Raw data were directly imported into the software. After mass detection, ADAP chromatogram builder functionality [35] was applied. The feature lists were processed as follows: first isotopic peaks were grouped with the pseudo-molecular ion, then a smoothing was performed in order to facilitate chromatogram deconvolution. The 40 feature lists coming from the CPC fractionation of one sample were aligned so that all the MS peak areas for one ion associated to its retention time are indicated on the same line. Finally, molecular formulae were assessed with the following conditions: $\mathrm{C}_{1-100} \mathrm{H}_{1-100} \mathrm{O}_{0-1} \mathrm{~S}_{0-1} \mathrm{~N}_{0-1}$ and a mass accuracy of $2 \mathrm{ppm} .[\mathrm{M}]^{+\bullet}$ were the main formed ions. Based on the formulae list obtained, several properties could be calculated such as the double bound equivalent (DBE) which represents the number of rings plus the number of double bonds. DBE values are calculated by the following equation for $\mathrm{C}_{c} \mathrm{H}_{h} \mathrm{O}_{0} \mathrm{~S}_{s} \mathrm{~N}_{n}$ compounds:

$$
D B E=\frac{2 c-h+n+2}{2}
$$

The processed data were exported in Excel software (Microsoft, USA) for further calculation as regards PAH semi-quantification. For each fraction, concentrations were calculated thanks to an external calibration. External calibration included pyrene, benz(a)anthracen, perylene, 
benzo(ghi)perylene and coronene. All compounds with a DBE of 12 were quantified with pyrene calibration curve. Benz(a)anthracene was used to quantify compounds with a DBE of 13 and 14; perylene for DBE of 15 and 16; benzo(ghi)perylene for DBE of 17 and 18 and coronene for DBE of 19 and 20. Only fractions with a concentration greater than $5 \mathrm{mg} / \mathrm{L}$ were quantified. Two calibration ranges were prepared: the first ranging from 5 to $100 \mathrm{mg} / \mathrm{L}$ and the second up to $500 \mathrm{mg} / \mathrm{L}$. Compounds with a concentration below $100 \mathrm{mg} / \mathrm{L}$ were quantified with the first range, while the second range was used for those with a concentration above $100 \mathrm{mg} / \mathrm{L}$. Then, the concentrations were converted in amount of analytes in each fraction. Finally, by summing the quantities of analytes in all the fractions, the total concentrations in the VGO could be measured. Taking into account the limit of quantification of $5 \mathrm{mg} / \mathrm{L}$ in the CPC fractions and that one compound eluted in around five CPC fractions, the limit of quantification in VGOs could be estimated to $10 \mathrm{mg} / \mathrm{kg}$.

The repeatability and reproducibility of the semi-quantitative method were evaluated by repeating three times the VGO 1 analysis: two times the same day and operator, and the last time at several weeks interval with a different operator. The averages of the relative standard deviations (RSD) for all the DBE families were 8 and $11 \%$ for repeatability and reproducibility respectively. Thus, the confidence interval for PAH quantification was $\pm 30 \%$.

\section{Results and discussion}

\subsection{Quantification methodology}

In this study, in order to apply our semi-quantitative three-dimensional method to a pool of samples, some points must be validated. The first one concerned the recovery efficiency of the off-line method as an evaporation step occurring between the CPC fractionation and the SFC analysis could lead to some losses of sample. The second one was about the quantification of alkylated PAHs. It is well known that VGOs contain hydrocarbons which could have up to 50 carbon atoms [36-38]. To quantify these more or less alkylated compounds, their response factors had to be compared to their non-alkylated homolog compounds. 
A solution of anthracene, pyrene, perylene, benzo(ghi)perylene and coronene was prepared at $c a$.

$50 \mathrm{mg} / \mathrm{L}$ for each compound using toluene as solvent. Five $\mathrm{mL}$ of this solution were injected into CPC.

The complete analytical method was applied including the evaporation step. MS data were processed as explained in the experimental part. Concentrations of standards in the 40 fractions were calculated thanks to an external calibration. Based on these concentrations, the amounts of standards in the fractions were calculated and summed. MS quantification showed recovery yields (82\%) might be due to a loss of standard during the evaporation of CPC fractions.

Based on these results, only PAHs with at least 4 rings (i.e. pyrene) were quantified in VGOs.

\subsubsection{Study of alkylated PAH standards}

Seven alkylated pyrenes including methyl and dimethylpyrene substituted at different positions, ethyl, propyl and butylpyrene as well as methylcoronene were selected to study the impact of the alkylation on the MS response (Figure 2). One $\mu \mathrm{L}$ of each of them at $200 \mathrm{mg} / \mathrm{L}$ was injected in SFC/HRMS.

Mass spectra of pyrene and alkylated pyrenes are presented in Figure 3. Mass spectrum of pyrene shows $[\mathrm{M}]^{+\bullet}$ ion whereas alkylated pyrenes form additional ions coming from in-source fragmentation of $[\mathrm{M}]^{+\bullet}$. Indeed, $[\mathrm{M}]^{+\bullet}$ ions are less stable than $[\mathrm{M}+\mathrm{H}]^{+}$ions and will therefore fragment more easily. The tendency of compounds to form $[\mathrm{M}]^{+\bullet}$ or $[\mathrm{M}+\mathrm{H}]^{+}$ions depends on the nature of the molecule but also on the experimental conditions used and in particular the nature of the dopant in the case of APPI. The ionisation of analytes via a dopant can take place either by charge or proton transfer. To favour the proton transfer in order to form predominantly $[\mathrm{M}+\mathrm{H}]^{+}$ions, the dopant must have both a low ionisation energy (IE) and a low proton affinity (PA). It was demonstrated in previous works that anisole provides better sensitivity than toluene $[29,39,40]$. Anisole has an IE of $8.20 \mathrm{eV}$ compared 
with $8.83 \mathrm{eV}$ for toluene [41]. Both dopants have a lower IE than the photon energy of the krypton lamp and higher than IE of PAHs. Thus, they can ionise our compounds of interest by charge transfer. On the other hand, PA of anisole is higher than that of toluene or acetone. Thus, the latter two dopants would be likely to promote proton transfer. However, the use of toluene as a dopant did not result in the formation of $[\mathrm{M}+\mathrm{H}]^{+}$ions during SFC/HRMS analysis. The very low IE of PAHs favours charge transfer and it will therefore be difficult to find conditions for $[\mathrm{M}+\mathrm{H}]^{+}$ion formation. Moreover, it is preferable to form only one type of ion rather than a mixture of radical and proton ions. The instability of $[\mathrm{M}]^{+\bullet}$ ions coming from alkylated PAHs leads to in-source fragmentation which might not be avoid. Since the desolvation conditions are relatively mild (APPI temperature $<350^{\circ} \mathrm{C}$ and ion transfer capillary temperature at $200^{\circ} \mathrm{C}$ ), modifying them would probably not completely prevent fragmentation.

For methyl and dimethylpyrenes, the most intense ion remain $[\mathrm{M}]^{+\bullet}$ while for pyrene with a longer alkyl chain (ethyl, propyl or butyl), $m / z 215$ which corresponds to $\left[\mathrm{M}-\mathrm{CH}_{3}\right]^{+},\left[\mathrm{M}-\mathrm{C}_{2} \mathrm{H}_{5}\right]^{+}$or $\left[\mathrm{M}-\mathrm{C}_{3} \mathrm{H}_{7}\right]^{+}$ ions becomes the most intense one. This ion is also visible on the mass spectra of methylpyrenes and dimethylpyrenes as $[\mathrm{M}-\mathrm{H}]^{+}$and $\left[\mathrm{M}-\mathrm{CH}_{3}\right]^{+}$ions respectively, but in a smaller extend. Finally, $[\mathrm{M}-\mathrm{H}]^{+}$ ions are also formed during the ionisation of the dimethylpyrenes. All the mass spectra are different, even for two isomers, the relative intensities of each ion are different. This uniqueness is a great source of information and allows to distinguish the kind of alkylation (i.e. dimethylpyrene versus ethylpyrene). However, it leads to a more complex quantification and especially for data processing.

External quantification with individual standards is not possible due to the lack of available commercial standards and the fact that thousands of compounds are present in a VGO sample. Thus, in this work, we have decided to semi-quantify PAHs using one standard per DBE family. For example, pyrene family has a DBE of 12 , so all compounds with the same DBE will be quantified using the calibration curve of pyrene. However, to apply this methodology, MS responses of alkylated PAHs have been evaluated and compared to the corresponding non-alkylated PAHs. As said previously, 
alkylated PAHs showed multiple ions. Two main ways are possible to take into account all the ions. The first one (Method\#1) consists in quantifying individually each ion using the pyrene calibration curve and then summing the calculated concentrations. The second way (Method\#2) consists in first summing the areas of each extracted ion chromatograms (EIC) of the different ions and second calculating the global concentration of the alkylated pyrene. The two methods were applied and the results are given in Table 4. Both methods gave quantifications with errors smaller than $10 \%$ except for 2,7-dimethylpyrene. Most of the time Method\#1 had better accuracy than Method\#2. Finally, alkylated pyrenes were also quantified using UV detection and the UV calibration curve of pyrene (Table 5). Despite the selection of the wavelength allowing the maximum response for each compound, significant biases were observed by systematically underestimating actual concentrations.

In conclusion, for the rest of the study Method\#1 using mass spectrometry was employed to semiquantify PAHs in VGO samples. Moreover, the experiences conducted on commercially available alkylated PAHs showed that the response factors of alkylated PAHs and their corresponding nonalkylated PAHs were relatively closed (Table S1). Thus, the alkylated PAHs could be semi-quantified using the response factors of the corresponding non-alkylated PAHs. Then, the calibration curves of several standards showed that PAHs with the same DBE had similar response factors. Figure S1 illustrated the example of chrysene and benz[a]anthracene which both have a DBE of 13 . Finally, based on the hypothesis, supported by experimental data, that alkylated PAHs as well as all PAHs with the same DBE have a similar response coefficient, we proposed to semi-quantified PAHs using the response factor of one standard for each DBE family. Although this approach has its own limitations, it can allow to have access to valuable data to compare different VGOs, as well as to monitor the evolution of PAHs formation during a process. All compounds with a DBE of 12 were quantified with pyrene calibration curve. Benz(a)anthracene was used to quantified compounds with a DBE of 13 and 14; perylene for DBE of 15 and 16; benzo(ghi)perylene for DBE of 17 and 18 and coronene for DBE of 19 and 20. A possible error on the quantification of alkylated PAHs may be done. 
The error will be more in the direction of overestimation in view of the error made on 2,7dimethylpyrene. However, these errors will be identical between the different samples since the matrix effects were limited by the CPCXSFC hyphenation. Thus, inter-sample comparisons and monitoring of $\mathrm{PAH}$ concentrations during a HCK process remain reliable.

\subsection{Analysis of VGOs by CPCXSFC/HRMS}

The six VGOs described in Table 1 were analysed using the CPCXSFC/HRMS method, providing a 2D plot specific to each sample (Figure 4). These 2D plots are fingerprints of the samples and revealed differences even for the same group of VGOs as for the four SRVGOs (from VGO 1 to 4). The use of high-resolution mass spectrometry enabled us to determine a possible molecular formula for each peak reported on the 2D plots, making it possible to distinguish PAHs and heteroelement-containing species. Nitrogen contents of the investigated samples varied from 0.10 to $0.35 \mathrm{wt} \%$, corresponding to 'neutral' or 'basic' nitrogen compounds whose structures were based on respectively an acridine or a carbazole core. When a classical analytical approach is employed involving a non-structural informative detector such as flame ionisation detector for gas chromatography, nitrogen species have to be removed prior quantitative analysis because they tend to co-elute with hydrocarbons. According to CPCXSFC maps illustrated in Figure 4, nitrogen-containing compounds from VGOs were specifically eluted in the first CPC fractions as they were partitioned mainly in the CPC mobile phase enriched in acetonitrile; and in the second part of the SFC chromatograms thanks to the interaction between polar groups of the stationary phase and nitrogen atoms of analytes (red boxes in Figure 4). Using (+)APPI, between 110 for VGO 1 and 510 for VGO 5 unique molecular features could be identified as $\mathrm{C}_{x} \mathrm{H}_{\mathrm{y}} \mathrm{N}$ without considering multiple isomers eluting at several retention times. At the opposite of nitrogen-containing compounds, PAHs were detected all along the CPC run (black boxes). Thus nitrogen compounds and hydrocarbons were directly and easily differentiated thanks to the CPCXSFC orthogonal separation conditions. Concerning sulphur-containing compounds, they are usually present in VGOs as alkyl and polybenzothiophenes. In the studied samples sulphur contents 
covered a large range of values from 0.30 to $2.58 \mathrm{wt} \%$ with between 210 for VGO 1 and 570 for VGO 3 identified chemical formulae. In our analysis conditions, sulphur-containing compounds were eluted all along the CPC run (i.e. 10-40 min) within the same chromatogram areas than the PAHs ones (black boxes). Indeed, aromatic sulphur-containing compounds have close chemical properties to aromatic compounds leading to similar retention in CPC and SFC. For example, pyrene $\left(\mathrm{C}_{16} \mathrm{H}_{10}\right.$, DBE 12) and dibenzothiophene $\left(\mathrm{C}_{16} \mathrm{H}_{10} \mathrm{~S}\right.$, DBE 12) were both eluted between 13 and $14 \mathrm{~min}$ in CPC and at 6.5 and 7.9 min respectively in SFC, while benzocarbazole or aminopyrene $\left(\mathrm{C}_{16} \mathrm{H}_{11} \mathrm{~N}\right.$, DBE 12) were eluted between 7 and $8 \mathrm{~min}$ in CPC and between 13.8 and $14.6 \mathrm{~min}$ in SFC. So in this case, the small difference in retention time between PAHs and sulphur compounds did not allow a quantification with UV detection, but it was sufficient to distinguish them in HRMS and avoid matrix effect in the ionisation source [29]. Thus, a specific treatment of HRMS data was performed to make a distinction between sulphur-containing molecules and PAHs, resulting in 2D plots dedicated only to hydrocarbons $\left(\mathrm{C}_{x} \mathrm{H}_{\mathrm{y}}\right.$ compounds) as shown in Figure 5 . As hydrocarbons were eluted in SFC separation according to their unsaturation level [29], SFC retention times were used to subdivide 2D plots as a function of PAH DBE. Then it was possible to get well-organised CPCXSFC chromatograms, providing a relevant structuration of maps based on DBE values, ranging from DBE inferior to 12 up to 19 and more. Such a 2D space organisation would be undoubtedly useful to describe the chemical composition of VGOs. It should be noticed that the intensities on the 2D plots were relative, so the direct comparison of peak intensities between samples was not possible. To go further, a semiquantification methodology previously set up was applied to the six samples and PAH contents were measured for each VGO from DBE 12. Although PAHs with a DBE lower than 12 were not quantified due to losses during the evaporation step, 2D plots in Figure 5 showed a large number of peaks in VGO 1 as well as in VGO 6, while the area dedicated to this DBE class was almost empty in VGO 5.

PAH contents measured for the six investigated samples from the CPCXSFC/HRMS analyses are reported in Table 6. Globally PAH contents decreased with DBE values and were scattered in a large scale depending on both the DBE value and the VGO. For instance PAH contents varied from 6296 
(VGO 3) to $14357 \mathrm{mg} / \mathrm{kg}$ (VGO 1) when considering a DBE value equal to 12 . All samples contained

333 PAHs at significant levels for DBE comprised between 12 and 17 but fewer contained PAHs with a DBE equal to 18 (high density samples do, like VGOs 4, 5 and 6) and only one sample, VGO 5 coming from a deep conversion process, still had PAHs with a DBE of 20.

The three-dimensional CPCXSFC/HRMS approach used in this study offered a detailed and semiquantitative description of PAH composition for VGOs. Thanks to the high resolution provided by the bidimensional chromatography and by mass spectrometry, it was possible to get more information about PAHs present in VGOs including data about their alkylation level. Among the set of VGOs investigated in this work, two different samples were selected as examples: a medium density straight-run vacuum gas oil (VGO 3) and a vacuum gas oil coming from a deep conversion process (VGO 5). For each sample, DBE versus the number of carbon atoms (nC) was plotted (Figure 6), reporting PAH contents thanks to the dot size. Such representation is classically used to describe complex products and have to be usually considered from a qualitative point of view due to matrix effects preventing quantification when the sample is introduced into HRMS analyser through direct infusion without any preliminary chromatographic separation [19]. However in our case, the use of bidimensional CPCXSFC enabled a reduction of such matrix effects [29] and provided a semiquantification of alkyl PAHs, assuming that all alkyls had the same response factors as their non-alkyl PAH reference. Therefore dots plotted in Figure 6 represent contents of PAHs and not HRMS signal intensities, so compositions of VGOs can be directly compared with each other. For instance PAH alkylation levels notably depended on both the DBE and the kind of VGO: PAHs with low DBE and short $\mathrm{CH}_{2}$ chains were mainly observed for the straight-run vacuum gas oil VGO 3 whereas PAHs were distributed over a broad range of DBE with rather long or numerous alkyl chains for deep conversion vacuum gas oil VGO 5. From the CPCXSFC/HRMS data, it was also possible to calculate a 'weighted average number of carbon atoms' $(\mathrm{nC})$ by weighting the number of carbon atoms of all the alkyl PAHs belonging to the same DBE group by the content of each hydrocarbon. Plotted as dotted lines in 
aromatic rings and in the alkyl chains and may be considered as a gravity center dedicated to each DBE group.

Evolution of the weighted average $\mathrm{nC}$ according to DBE values for the six investigated VGOs were illustrated in Figure 7. For comparison PAHs having no alkyl substituent (i.e. pyrene, benz(a)anthracene, perylene, benzo(ghi)perylene and coronene) were also plotted as references in Figure 7. It appears clearly that the gap in terms of weighted average $\mathrm{nC}$ between the PAHs in VGOs and the non-alkyl references is higher for low DBE and tends to be alleviated for increasing DBE. For DBE 12, the non-alkyl reference, pyrene, is made of 16 carbon atoms whereas in VGO samples the weighted average $\mathrm{nC}$ was comprised between 22 (VGO 3) and 24 (VGO 5). Thus, compounds with a pyrene core were alkylated with on average 8 carbon atoms for VGO 5. For PAHs with a higher DBE, the alkylation level was lower with, for example, two carbon atoms in average in the alkyl chain for compounds with a DBE of 17 in VGO 6 (based on benzo(ghi)perylene as non-alkyl reference). This difference of alkylation degree between DBE could be directly observed on the 2D plots in Figure 5 . Indeed, PAHs with a DBE $\geq 17$ were eluted only in the first part of CPC while PAHs with a DBE between 12 and 14 were eluted all along the CPC run until the extrusion of the stationary phase. As already discussed from Table 6, the sample VGO 5 coming from a deep conversion process exhibited a special composition when compared to straight-run vacuum gas oils: in addition to contain heavy PAHs, VGO 5 presented a more important alkylation degree than the other VGOs. All the graphs representing the concentration as a function of $\mathrm{nC}$ for DBE 12 to 20 are available in Figure S2.

Finally CPCXSFC/HRMS analysis allowed a more accurate characterisation than an analysis by SFC/HRMS. As shown previously [20], a strong ionisation suppression can occur during VGO analysis by SFC/HRMS due to ionisation competition of analytes entering simultaneously in the ionisation source [29]. By calculating the weighted average $\mathrm{nC}$ based on the intensity of ions detected in SFC/HRMS, it appeared that the average $\mathrm{nC}$ for a given DBE was higher than the one calculated using data from CPCXSFC/HRMS analysis. As an example, for compounds with a DBE of 12 detected in 
VGO 4, the average $\mathrm{nC}$ measured by SFC/HRMS was about 37 , while this latter was only 23 by $\mathrm{CPC} \times \mathrm{SFC} / \mathrm{HRMS}$. Therefore, highly alkylated compounds seemed to be preferentially ionised over less alkylated analytes, which created a bias in the characterisation of VGOs by SFC/HRMS. The hyphenation of CPC and SFC/HRMS was therefore required to achieve a more accurate characterisation, reflecting the composition of the VGOs.

In addition, in the first part of this study, we have demonstrated that mass spectra of alkylated PAHs were different according to the alkyl chain type. In this way, dimethylpyrene and ethylpyrene can be differentiated for instance. As an example, mass spectra of main compounds belonging to the pyrene family (DBE 12) in VGO 6 with one, two and three carbon atoms in the alkyl chain are presented in Figure S3. For all of them, $[\mathrm{M}]^{+\bullet}$ were the main ion meaning that instead of an alkyl chain such as ethyl or propyl groups, carbon atoms belonged to several methyl groups. This kind of information about isomerisation of the alkyl chains should be very useful to better understand the role played by PAHs in the VGO reactivity during HDT and HCK processes.

\subsection{Evolution of PAH concentrations during hydrotreating and hydrocracking of a VGO}

In the previous section, PAHs were quantified in several VGOs. In this section, VGO 2 was selected and the by-products from the HDT and HCK of this feedstock were characterized in terms of PAH concentrations in order to have a global view of their evolution. Monitoring of PAH contents was performed on several products coming from single-stage HCK in a recycling stream configuration (Figure 8). HDT- $\mathrm{t}_{0}$ was collected after the HDT of the feed while the unconverted oil samples (UCO- $\mathrm{t}_{\mathrm{i}}$ ) were collected from the atmospheric distillation residue of HCK products with an initial boiling point temperature superior to $370^{\circ} \mathrm{C}$.

These HCK products were collected under several process conditions: $1 /$ HDT- $t_{0}$ and UCO- $t_{0}$ were collected before the recycle flow was started (once-through configuration), 2 / UCO- $t_{1}$ to UCO- $t_{5}$ were sampled once UCO coming from the HCK step was recycled and introduced at the inlet of the HDT 
reactor in co-processing with non-hydrotreated VGO 2 feedstock. Table 2 summarises the

408 experimental conditions under which the samples were obtained.

409

The three-dimensional analytical methodology was applied to VGO 2 while SFC/HRMS could be used for semi-quantification of PAHs in HCK products as they did not contain, or in a small extend, sulphur and nitrogen compounds. Thus, the SFC and HRMS dimensions were both used for the analysis of VGO and by-products, making data comparison easier.

\subsubsection{Single-stage once-through configuration of hydrocracking}

A product resulting from the HDT of VGO 2 was first collected as illustrated in Figure 8 and was referred as HDT-t $t_{0}$ sample. In a second time, it was processed in a HCK reactor to convert its heavy fraction (i.e. compounds having a boiling point temperature superior to $370^{\circ} \mathrm{C}$ ) in lighter valuable distillation cuts which are naphta, kerosene and atmospheric gas oil. The vacuum residue with an initial boiling point temperature superior to $370^{\circ} \mathrm{C}$, also called $U C O$, was obtained from the distillation of the HCK products. PAH contents were measured in the three samples: the VGO before HDT (VGO 2 sample), the liquid after HDT and prior HCK (HDT- $\mathrm{t}_{0}$ ) and the vacuum residue after HCK (UCO- $\left.\mathrm{t}_{0}\right)$. For comparison of the PAH contents, the distillation yield of the UCO was taken into account to calculate normalised PAH contents (referred as UCO- ${ }_{0}{ }^{*}$ ) that could be directly compared to the contents in VGO 2 and HDT-t $t_{0}$ samples.

Evolution of PAH contents of VGO 2, HDT- $\mathrm{t}_{0}$ and UCO- $\mathrm{t}_{0}{ }^{*}$ samples according to DBE values are reported in Figure 9A. At first, we observed a clear drop of the contents during HDT for PAHs having a DBE comprised between 12 and 16, indicating a deep hydrogenation of these aromatic species in the investigated process conditions. For example, the concentration of compounds with a DBE of 12 was divided by 4 between VGO 2 and HDT- $\mathrm{t}_{0}$ samples. Similarly, during HCK a decrease in the concentration of compounds with a DBE between 12 and 16 was observed, but in a smaller extent compared to the decrease that occurred during HDT. The concentration of compounds with a DBE of 
12 was this time divided by a factor smaller than 3 . As regards PAHs with DBE superior to 17 , no significant change could be observed due to the low concentrations measured (below $10 \mathrm{mg} / \mathrm{kg}$ ).

Moreover, evolution of the weighted average $\mathrm{nC}$ according to DBE values is illustrated in Figure $9 \mathrm{~B}$. Initially, VGO 2 contained PAHs with a decrease of the alkylation degree with the DBE value by considering the gap between the weighted average $\mathrm{nC}$ from PAHs in VGO 2 and the non-alkyl references. During HDT, the weighted average $\mathrm{nC}$ increased for DBE 12, 13 and 14 between VGO 2 and HDT- $\mathrm{t}_{0}$. This observation may indicate that PAHs with a low level of alkylation were preferably hydrotreated. For DBE from 15 to 18, this observation could not be done since PAHs already present in the feed were only slightly alkylated. On the opposite, the alkylation level was rather constant in the UCO- $\mathrm{t}_{0}{ }^{*}$ sample whatever the DBE value was. Indeed, the gap between the weighted average $\mathrm{nC}$ measured for PAHs in UCO- $\mathrm{t}_{0}{ }^{*}$ and non-alkyl references was comprised between one and three for all DBE values. The strong decrease of the weighted average $\mathrm{nC}$ between HDT- $\mathrm{t}_{0}$ and UCO- $\mathrm{t}_{0}$ * samples for DBE 12, 13 and 14 was the consequence of cracking reactions of highly alkylated PAHs. These semi-quantitative results obtained from three selected examples show that the content and the composition of PAHs were both specific to a kind of sample. For each DBE value, it was possible to plot the distribution of PAH contents according to $\mathrm{nC}$; for instance such distributions measured for DBE equal to 12 were illustrated in Figure 9C. The number of carbon atoms for VGO 2 and HDT-to samples was spread between 18 and 35; whereas, after HCK, nC was only distributed between 18 and 23 for UCO- $\mathrm{t}_{0}{ }^{*}$ sample. Also, the drop of PAH content between VGO 2 and HDT- $\mathrm{t}_{0}$ samples was more important for the lowest $\mathrm{nC}$ with for example a drop of a 13 times for PAHs with 19 carbon 451 atoms, while the global PAH content for DBE 13 decreased with a factor of only 4. Although we observed a decrease of the concentration between HDT- $\mathrm{t}_{0}$ and UCO- $\mathrm{t}_{0} *$ samples for PAHs with a DBE of 13 from Figure 9A, Figure $9 \mathrm{C}$ showed an increase of PAH contents with $\mathrm{nC}$ between 19 and 21, and slightly alkylated ones. 
Finally, during single-stage once-through hydrocracking, the HDT of the feed lead to a decrease in the PAH concentrations due to deep hydrogenation of double bonds $\mathrm{C}=\mathrm{C}$. It seemed that PAHs with a low alkylation level were preferably hydrogenated. Then, HCK allowed to convert highly alkylated PAHs into PAHs with a low level of alkylation.

\subsubsection{Single-stage with recycle configuration of hydrocracking}

The analytical methodology was also applied to some products collected once a part of the UCO from the HCK step and following distillation was used as co-processing feedstock together with the initial VGO 2 and was introduced into the HDT reactor (single-stage HCK configuration). In addition to UCO$t_{0}$ sample collected before the start-up of the recycle stream, five UCO collected under different process conditions were investigated and referred as UCO- $t_{1}, U C O-t_{2}, U C O-t_{3}, U C O-t_{4}$, and UCO- $t_{5}$ samples in relation to their increasing time on stream. As reported in Table 2, products at the outlet of HCK reactor exhibited quite similar amounts of high boiling point compounds (ca. $47-50 \mathrm{wt} \%$ of $370^{\circ} \mathrm{C}+$ fraction)

Contents of the PAHs contained in the HCK vacuum residues were measured using the SFC/HRMS approach and normalised taking into account the yield of the $370^{\circ} \mathrm{C}+$ cut (see Table 2 for the yields). These contents were referred as UCO- $\mathrm{t}_{\mathrm{i}}{ }^{*}$ and were expressed as $\mathrm{mg} / \mathrm{kg}$ of feed, considering both VGO 2 and the recycle stream as feedstocks in the present co-processing configuration. Evolutions of the PAH contents for VGO 2 and UCO- $\mathrm{t}_{\mathrm{i}}{ }^{*}$ samples are represented as a function of DBE values in Figure 10. For DBE inferior to 16, PAH contents of HCK products were all lower than the ones measured for VGO 2 whatever was the UCO recycle flow rate: these PAH contents first drastically dropped from the VGO 2 feedstock to the first HCK product (UCO- $\mathrm{t}_{0}{ }^{*}$ ) due to the hydrogenation of the aromatic rings; then they increased progressively with time on stream, indicating an accumulation of PAHs in the HCK reactor. For compounds with DBE $\geq 17$ (i.e. PAHs with six aromatic rings at minimum), their contents became higher than in the VGO once UCO recycle stream had been started. This accumulation of PAHs tend to increase from UCO- $\mathrm{t}_{0}{ }^{*}$ to $\mathrm{UCO}-\mathrm{t}_{5}{ }^{*}$. 
482 illustrated in Figure 11A. As discussed previously, VGO 2 contained PAHs with an alkylation degree which decreases with the DBE value. On the opposite to VGO 2, HCK products were made of PAHs with a rather low and constant alkylation degree whatever the DBE value was. Moreover, the weighted averages $\mathrm{nC}$ were similar for all the $\mathrm{HCK}$ products and were particularly lower than the ones measured for PAHs in VGO 2 when considering DBE values inferior to 15 . These results show that, under our conditions, PAHs with a low DBE were significantly affected by hydrogenation at the first stage of HCK and the alkyl structures still remained the same even when PAH contents strongly increased with time on stream, so the same kind of PAHs were accumulated with time in the HCK reactor as illustrated in Figure 11B for compounds with a DBE of 13.

491

492

493

494

495

Although the aim of this study was not to clearly identify the impact of each process parameter on PAH concentrations, evolution of PAHs according to R2 temperature was plotted in Figure 12. Absolute values of R2 temperatures could not be given due to confidential nature. Figure 12 highlighted the impact of R2 temperature on the PAH concentration. Indeed, R2 temperature is usually increased in order to compensate the catalyst deactivation and keep a constant conversion. However, it seemed that this increase in temperature also leads to an increase of PAH concentration. For PAHs with 4 and 5 rings (DBE between 12 and 16), the concentrations range from $1581 \mathrm{mg} / \mathrm{kg}$ at $\mathrm{T}_{0}$ to $7717 \mathrm{mg} / \mathrm{kg}$ at $\mathrm{T}_{+30^{\circ} \mathrm{C}}$ which represents an increase of $390 \%$. For PAHs with at least 6 rings (DBE $\geq 17)$, this increase was even more important by going from 88 to $1216 \mathrm{mg} / \mathrm{kg}$ or an increase of more than $1000 \%$.

\section{Conclusion}

In this study we have successfully managed to semi-quantify PAHs in vacuum gas oils using a threedimensional off-line CPCXSFC/HRMS approach while chemical compositions of several HDT/HCK products coming from once-through or recycle HCK configurations were described by SFC/HRMS. As regards analysis of VGOs, the use of an orthogonal CPCXSFC configuration with an enhanced 
resolution capacity enabled a clear separation of hydrocarbons and nitrogen-containing compounds. Moreover a specific treatment of high-resolution mass spectrometric data was performed to differentiate PAHs from sulphur-containing compounds prior to their quantification. As a result, wellorganised CPCXSFC chromatograms provided a relevant separation of PAHs according to both unsaturation level (DBE) and alkylation degree. This dedicated methodology allowed the semiquantification of PAHs having DBE superior to 12. Concerning the HDT/HCK products, their sulphur and nitrogen contents were low enough to make possible semi-quantification of PAHs directly by SFC/HRMS. PAH contents tend to decrease during the HDT of VGO, PAHs with a low alkylation level being preferably hydrogenated. Then HCK reaction allowed to cut alkyl chains and convert highly alkylated PAHs into PAHs with a low level of alkylation. An accumulation of PAHs and especially of PAHs with more than six aromatic rings occurred when an UCO recycle stream was applied. Furthermore our results have shown that the temperature of HCK unit was correlated with the PAH concentration. As a conclusion, this work has demonstrated that reliable and relevant semiquantitative data about PAH compositions in VGOs and their HDT/HCK products can be achieved thanks to our CPCXSFC/HRMS methodology. Such useful data will be crucial for a better understanding of PAH formation and a further powerful modelling.

\section{Acknowledgments}

Authors would like to greatly thank Luc Davy and Benoit Celse from IFP Energies Nouvelles for the precious discussions about processes and samples. Authors also thank Alexandra Berlioz-Barbier for her advices on MS data treatment.

\section{References}

[1] A. Demirbas, A. Bafail, A.-S. Nizami, Heavy oil upgrading: Unlocking the future fuel supply, $\begin{array}{llllll}\text { Petroleum Science } & \text { and } & \text { (2016) 303-308. }\end{array}$ https://doi.org/10.1080/10916466.2015.1136949. 
530 [2] J. Ancheyta, M.S. Rana, E. Furimsky, Hydroprocessing of heavy petroleum feeds: Tutorial, 531 Catalysis Today 109 (2005) 3-15. https://doi.org/10.1016/j.cattod.2005.08.025.

532 [3] G. Valavarasu, M. Bhaskar, K.S. Balaraman, Mild Hydrocracking-A Review of the Process, 533 Catalysts, Reactions, Kinetics, and Advantages, Petroleum Science and Technology 21 (2003) 1185-1205. https://doi.org/10.1081/LFT-120017883.

[4] M. Sau, K. Basak, U. Manna, M. Santra, R.P. Verma, Effects of organic nitrogen compounds on hydrotreating and hydrocracking reactions, Catalysis Today 109 (2005) 112-119. https://doi.org/10.1016/j.cattod.2005.08.007.

[5] C. Peng, Z. Cao, Y. Du, R. Zeng, R. Guo, X. Duan, X. Fang, Optimization of a Pilot Hydrocracking Unit To Improve the Yield and Quality of Jet Fuel Together with Heavy Naphtha and Tail Oil, Ind. Eng. Chem. Res. 57 (2018) 2068-2074. https://doi.org/10.1021/acs.iecr.7b04981.

[6] O.V. Klimov, K.A. Nadeina, P.P. Dik, G.I. Koryakina, V. Pereyma, M.O. Kazakov, S.V. Budukva, E. Gerasimov, I.P. Prosvirin, D.I. Kochubey, A.S. Noskov, CoNiMo/Al2O3 catalysts for deep hydrotreatment of vacuum gasoil, Catalysis Today 271 (2016) 56-63. https://doi.org/10.1016/j.cattod.2015.11.004.

546

[7] C.S. Hsu, P.R. Robinson, Practical Advances in Petroleum Processing: Volume 1, Springer, New York, 2009.

[8] R.F. Sullivan, M.M. Boduszynski, J.C. Fetzer, Molecular transformations in hydrotreating and hydrocracking, Energy Fuels 3 (1989) 603-612. https://doi.org/10.1021/ef00017a013.

[9] J.C. Fetzer, THE CHEMISTRY AND ANALYSIS OF LARGE PAHs, Polycyclic Aromatic Compounds 27 (2007) 143-162. https://doi.org/10.1080/10406630701268255.

[10] K. Basak, M. Sau, U. Manna, R.P. Verma, Industrial hydrocracker model based on novel continuum lumping approach for optimization in petroleum refinery, Catalysis Today 98 (2004) 253-264. https://doi.org/10.1016/j.cattod.2004.07.056. 
554 [11] P.J. Becker, B. Celse, D. Guillaume, H. Dulot, V. Costa, Hydrotreatment modeling for a variety of VGO feedstocks: A continuous lumping approach, Fuel 139 (2015) 133-143. https://doi.org/10.1016/j.fuel.2014.08.032.

557

[12] C. Fan, J. Long, Effect of Feed Properties on Product Distribution from the Modeling of an Industrial Hydrocracking Process, Pet. Chem. 60 (2020) 174-180. https://doi.org/10.1134/\$0965544120020024.

560

561

562

563

564

565

566

567

568

569

570

571

572

573

574

575

576

577

578

[13] H.M. Lababidi, D. Chedadeh, M.R. Riazi, A. Al-Qattan, H.A. Al-Adwani, Prediction of product quality for catalytic hydrocracking of vacuum gas oil, Fuel 90 (2011) 719-727. https://doi.org/10.1016/j.fuel.2010.09.046.

[14] H. Puron, P. Arcelus-Arrillaga, K.K. Chin, J.L. Pinilla, B. Fidalgo, M. Millan, Kinetic analysis of vacuum residue hydrocracking in early reaction stages, Fuel 117 (2014) 408-414. https://doi.org/10.1016/j.fuel.2013.09.053.

[15] H. Zhou, J. Lu, Z. Cao, J. Shi, M. Pan, W. Li, Q. Jiang, Modeling and optimization of an industrial hydrocracking unit to improve the yield of diesel or kerosene, Fuel 90 (2011) 3521-3530. https://doi.org/10.1016/j.fuel.2011.02.043.

[16] A. Pyhälahti, J. Kanervo, S. Kuitunen, Comprehensive Residual Oil Hydrocracking Reaction Kinetic Modeling Combined with Effective Phase Equilibrium Calculation, Ind. Eng. Chem. Res. 57 (2018) 11271-11279. https://doi.org/10.1021/acs.iecr.8b01834.

[17] P.J. Becker, B. Celse, D. Guillaume, V. Costa, L. Bertier, E. Guillon, G. Pirngruber, A continuous lumping model for hydrocracking on a zeolite catalysts: Model development and parameter identification, Fuel 164 (2016) 73-82. https://doi.org/10.1016/j.fuel.2015.09.057.

[18] A.G. Marshall, R.P. Rodgers, Petroleomics: The next grand challenge for chemical analysis, Acc. Chem. Res. 37 (2004) 53-59. https://doi.org/10.1021/ar020177t.

[19] A.G. Marshall, R.P. Rodgers, Petroleomics: Chemistry of the underworld, PNAS 105 (2008) 18090-18095. 
579 [20] H. Muller, N.A. Alawani, F.M. Adam, Innate Sulfur Compounds as an Internal Standard for 580 Determining Vacuum Gas Oil Compositions by APPI FT-ICR MS, Energy Fuels 34 (2020) 82608273. https://doi.org/10.1021/acs.energyfuels.0c01365.

582

583

584

585

586

587

588

589

590

591

592

593

594

595

596

597

598

599

600

601

602

[21] T.M. Schaub, R.P. Rodgers, A.G. Marshall, K. Qian, L.A. Green, W.N. Olmstead, Speciation of Aromatic Compounds in Petroleum Refinery Streams by Continuous Flow Field Desorption Ionization FT-ICR Mass Spectrometry, Energy Fuels 19 (2005) 1566-1573. https://doi.org/10.1021/ef049734d.

[22] J.M. Purcell, I. Merdrignac, R.P. Rodgers, A.G. Marshall, T. Gauthier, I. Guibard, Stepwise Structural Characterization of Asphaltenes during Deep Hydroconversion Processes Determined by Atmospheric Pressure Photoionization (APPI) Fourier Transform Ion Cyclotron Resonance (FTICR) Mass Spectrometry $\quad+$ Energy Fuels $24 \quad$ (2010) 2257-2265. https://doi.org/10.1021/ef900897a.

[23] R.P. Rodgers, M.M. Mapolelo, W.K. Robbins, M.L. Chacón-Patiño, J.C. Putman, S.F. Niles, S.M. Rowland, A.G. Marshall, Combating selective ionization in the high resolution mass spectral characterization of complex mixtures, Faraday Discuss. 218 (2019) 29-51. https://doi.org/10.1039/c9fd00005d.

[24] P.M. Lalli, J.M. Jarvis, A.G. Marshall, R.P. Rodgers, Functional Isomers in Petroleum Emulsion Interfacial Material Revealed by Ion Mobility Mass Spectrometry and Collision-Induced $\begin{array}{lllll}\text { Dissociation, } & \text { Energy } & \text { Fuels } & 31 & \text { (2017) 311-318. }\end{array}$ https://doi.org/10.1021/acs.energyfuels.6b02411.

[25] S.K. Panda, H. Muller, T.A. Al-Qunaysi, O.R. Koseoglu, Determination of heavy polycyclic aromatic hydrocarbons by non-aqueous reversed phase liquid chromatography: Application and limitation in refining streams, Journal of Chromatography A 1533 (2018) 30-37. https://doi.org/10.1016/j.chroma.2017.11.061. 
[26] M. Piparo, L. Flamant, G. Jousset, P. Cardinael, P. Giusti, Careful Investigations of PTV Injection Parameters for the Analysis of Vacuum Gas Oil by High-Temperature Comprehensive GC $\times$ GC, Energy Fuels 34 (2020) 12010-12017. https://doi.org/10.1021/acs.energyfuels.0c01314.

[27] T. Dutriez, M. Courtiade, D. Thiébaut, H. Dulot, F. Bertoncini, J. Vial, M.-C. Hennion, Hightemperature two-dimensional gas chromatography of hydrocarbons up to nC60 for analysis of vacuum gas oils, J. Chromatogr. A $1216 \quad$ (2009) 2905-2912. https://doi.org/10.1016/j.chroma.2008.11.065.

[28] L. Mahé, M. Courtiade, C. Dartiguelongue, J. Ponthus, V. Souchon, D. Thiébaut, Overcoming the high-temperature two-dimensional gas chromatography limits to elute heavy compounds, J. Chromatogr. A 1229 (2012) 298-301. https://doi.org/10.1016/j.chroma.2012.01.030.

[29] C. Reymond, A. Le Masle, C. Colas, N. Charon, Input of an Off-Line, Comprehensive, ThreeDimensional Method (CPCXSFC/HRMS) to Quantify Polycyclic Aromatic Hydrocarbons in Vacuum Gas Oils, Anal. Chem. 92 (2020) 6684-6692. https://doi.org/10.1021/acs.analchem.0c00605.

[30] N. Itoh, Y. Aoyagi, T. Yarita, Optimization of the dopant for the trace determination of polycyclic aromatic hydrocarbons by liquid chromatography/dopant-assisted atmospheric-pressure photoionization/mass spectrometry, Journal of Chromatography A 1131 (2006) 285-288. https://doi.org/10.1016/j.chroma.2006.08.091.

[31] T. Ghislain, P. Faure, R. Michels, Detection and monitoring of PAH and oxy-PAHs by high resolution mass spectrometry: comparison of ESI, APCI and APPI source detection, J Am Soc Mass Spectrom 23 (2012) 530-536. https://doi.org/10.1007/s13361-011-0304-8.

[32] A.K. Huba, K. Huba, P.R. Gardinali, Understanding the atmospheric pressure ionization of petroleum components: The effects of size, structure, and presence of heteroatoms, Sci. Total Environ. 568 (2016) 1018-1025. https://doi.org/10.1016/j.scitotenv.2016.06.044.

[33] J. Santos, M. Pudenzi, A. Wisniewski Jr., M. Breitkreitz, M. Eberlin, Optimization of Atmospheric Pressure Photoionization for the Crude Oil Analysis Using Ultra-High Resolution Mass Spectrometry, J. Braz. Chem. Soc. (2018). https://doi.org/10.21577/0103-5053.20180214. 
[34] T. Pluskal, S. Castillo, A. Villar-Briones, M. Orešič, MZmine 2: Modular framework for processing, visualizing, and analyzing mass spectrometrybased molecular profile data, BMC Bioinformatics (2010).

[35] O.D. Myers, S.J. Sumner, S. Li, S. Barnes, X. Du, One Step Forward for Reducing False Positive and False Negative Compound Identifications from Mass Spectrometry Metabolomics Data: New Algorithms for Constructing Extracted Ion Chromatograms and Detecting Chromatographic Peaks, Anal. Chem. 89 (2017) 8696-8703. https://doi.org/10.1021/acs.analchem.7b00947.

[36] S.K. Panda, N.A. Alawani, A.R. Lajami, T.A. Al-Qunaysi, H. Muller, Characterization of aromatic hydrocarbons and sulfur heterocycles in Saudi Arabian heavy crude oil by gel permeation chromatography and ultrahigh resolution mass spectrometry, Fuel 235 (2019) 1420-1426.

[37] E. Niyonsaba, J.M. Manheim, R. Yerabolu, H.I. Kenttämaa, Recent Advances in Petroleum Analysis by Mass Spectrometry, Anal. Chem. 91 (2019) 156-177. https://doi.org/10.1021/acs.analchem.8b05258.

[38] W. Wang, Y. Liu, Z. Liu, S. Tian, Detailed Chemical Composition of Straight-Run Vacuum Gas Oil and Its Distillates as a Function of the Atmospheric Equivalent Boiling Point, Energy Fuels (2016). https://doi.org/10.1021/acs.energyfuels.5b02803.

[39] D.R. Smith, D.B. Robb, M.W. Blades, Comparison of dopants for charge exchange ionization of nonpolar polycyclic aromatic hydrocarbons with reversed-phase LC-APPI-MS, J Am Soc Mass Spectrom 20 (2009) 73-79. https://doi.org/10.1016/j.jasms.2008.09.012.

[40] S.S.M. Amad, Z.A. Al-Talla, Multicomponent mixed dopant optimization for rapid screening of polycyclic aromatic hydrocarbons using ultra high performance liquid chromatography coupled to atmospheric pressure photoionization high-resolution mass spectrometry, Rapid Commun. Mass Spectrom. 26 (2012) 1488-1496. https://doi.org/10.1002/rcm.6250.

[41] P. ARPINO, Couplages chromatographiques avec la spectrométrie de masse. III, 2009 (accessed 26 October 2017). 


\section{Figure captions}

Figure 1: Scheme of the CPCXSFC/HRMS methodology

Figure 2: Alkylated PAHs investigated for the study of MS response factors

Figure 3: Mass spectra of pyrene and alkylated pyrenes

Figure 4: CPCXSFC/HRMS BPC plots of six VGO samples. PAHs and sulphur-containing compounds are included in the black boxes and nitrogen-containing compounds in the red boxes

Figure 5: CPCXSFC/HRMS BPC plots specific to hydrocarbons in six VGO samples. Colour boxes correspond to DBE (refer to plot F)

Figure 6: DBE as a function of $\mathrm{nC}$ for (A) VGO 3 and (B) VGO 5; dotted lines correspond to the weighted average $\mathrm{nC}$.

Figure 7: Weighted average number of carbon atoms for each DBE

Figure 8: Scheme of single-stage HCK in a recycling configuration

Figure 9: Evolution of (A) PAH contents for each DBE, (B) weighted average number of carbon atoms for each DBE and (C) PAH contents according to the number of carbon atoms for DBE 13 for VGO 2, HDT- $\mathrm{t}_{0}$ and $\mathrm{UCO}-\mathrm{t}_{0} *$ samples

Figure 10: Evolution of PAH contents for each DBE for VGO 2 and HCK products UCO- $\mathrm{t}_{0}$ * to UCO- $\mathrm{t}_{5}$ * Figure 11: (A) Evolution of weighted average number of carbon atoms for each DBE and (B) PAH contents according to the number of carbon atoms for DBE 13 for VGO 2 and HCK products UCO- $\mathrm{t}_{0}$ * to $\mathrm{UCO}-\mathrm{t}_{5} *$

Figure 12: R2 temperature according to $\mathrm{PAH}$ concentrations in $\mathrm{UCO}-\mathrm{t}_{\mathrm{i}}{ }^{*}$ samples

\section{$\underline{\text { Tables }}$}

Table 1: Physical and chemical properties of petroleum samples investigated in this work 


\begin{tabular}{|c|c|c|c|c|c|}
\hline Samples & Type & $\begin{array}{c}\text { Specific } \\
\text { gravity at } \\
15^{\circ} \mathrm{C}\left(\mathrm{g} / \mathrm{cm}^{3}\right)\end{array}$ & $\begin{array}{l}\text { Boiling points } \\
\text { at } 5 \text { and } 95 \% \\
\text { distilled }\left({ }^{\circ} \mathrm{C}\right)\end{array}$ & $\begin{array}{l}\text { Sulfur } \\
\text { content } \\
\text { (mg/kg) }\end{array}$ & $\begin{array}{l}\text { Nitrogen } \\
\text { content } \\
(\mathrm{mg} / \mathrm{kg})\end{array}$ \\
\hline & & ASTM D4052 & ASTM D2887 & ASTM D2622 & ASTM D4629 \\
\hline VGO 1 & SRVGO & 0.9208 & $265-509$ & 2974 & 1160 \\
\hline VGO 2 & SRVGO & 0.9211 & $342-561$ & 15351 & 1404 \\
\hline VGO 3 & SRVGO & 0.9244 & $352-544$ & 25792 & 982 \\
\hline VGO 4 & SRVGO & 0.9284 & $394-581$ & 18921 & 1395 \\
\hline VGO 5 & DCVGO & 0.9306 & $390-567$ & 6702 & 3510 \\
\hline VGO 6 & $\begin{array}{c}\text { SRVGO + DCVGO } \\
(50 / 50 \mathrm{v} / \mathrm{v})\end{array}$ & 0.9314 & $346-551$ & 10777 & 2610 \\
\hline
\end{tabular}

678

Table 2: Process parameters during UCO- $\mathrm{t}_{\mathrm{i}}$ sampling

\begin{tabular}{|c|c|c|c|c|}
\hline Samples & $\begin{array}{l}\text { Time on } \\
\text { stream } \\
\text { (h) }\end{array}$ & $\begin{array}{c}\mathrm{R} 1 \\
\text { temperature } \\
\left({ }^{\circ} \mathrm{C}\right)\end{array}$ & $\begin{array}{c}\mathrm{R} 2 \\
\text { temperature } \\
\left({ }^{\circ} \mathrm{C}\right)\end{array}$ & $\begin{array}{c}\text { Yield in } \\
370^{\circ} \mathrm{C}+\text { cut } \\
(w t \%)\end{array}$ \\
\hline HDT-t ${ }_{0}$ & \multirow{2}{*}{0} & \multirow{2}{*}{$\mathrm{T}_{0}$} & \multirow{2}{*}{$\mathrm{T}_{0}$} & \multirow{2}{*}{49.7} \\
\hline $\mathrm{UCO}-\mathrm{t}_{0}$ & & & & \\
\hline UCO-t ${ }_{1}$ & 583 & $\mathrm{~T}_{0}+3^{\circ} \mathrm{C}$ & $\mathrm{T}_{0}+10^{\circ} \mathrm{C}$ & 49.6 \\
\hline UCO- $\mathrm{t}_{2}$ & 847 & $\mathrm{~T}_{0}+3^{\circ} \mathrm{C}$ & $\mathrm{T}_{0}+22^{\circ} \mathrm{C}$ & 45.4 \\
\hline UCO- $t_{3}$ & 1015 & $\mathrm{~T}_{0}+7^{\circ} \mathrm{C}$ & $\mathrm{T}_{0}+25^{\circ} \mathrm{C}$ & 48.7 \\
\hline ucO-t $t_{4}$ & 1135 & $\mathrm{~T}_{0}+7^{\circ} \mathrm{C}$ & $\mathrm{T}_{0}+29^{\circ} \mathrm{C}$ & 45.8 \\
\hline UCO- $t_{5}$ & 1207 & $\mathrm{~T}_{0}+7^{\circ} \mathrm{C}$ & $\mathrm{T}_{0}+31^{\circ} \mathrm{C}$ & 47.2 \\
\hline
\end{tabular}

680

Table 3: Recovery yields of PAH standards analysed with the overall off-line CPCXSFC/HRMS method

\begin{tabular}{|c|c|c|c|}
\hline & $\begin{array}{c}\text { Amount of } \\
\text { standard injected } \\
\text { into CPC (mg) }\end{array}$ & $\begin{array}{c}\text { Total amount } \\
\text { measured by } \\
\text { SFC/HRMS (mg) }\end{array}$ & $\begin{array}{c}\text { Recovery } \\
\text { yield (\%) }\end{array}$ \\
\hline Anthracene & 0.231 & 0.189 & 82 \\
\hline Pyrene & 0.224 & 0.219 & 98 \\
\hline Perylene & 0.233 & 0.210 & 90 \\
\hline Benzo(ghi)perylene & 0.238 & 0.220 & 93 \\
\hline Coronene & 0.231 & 0.224 & 97 \\
\hline
\end{tabular}

682 
with two different methods

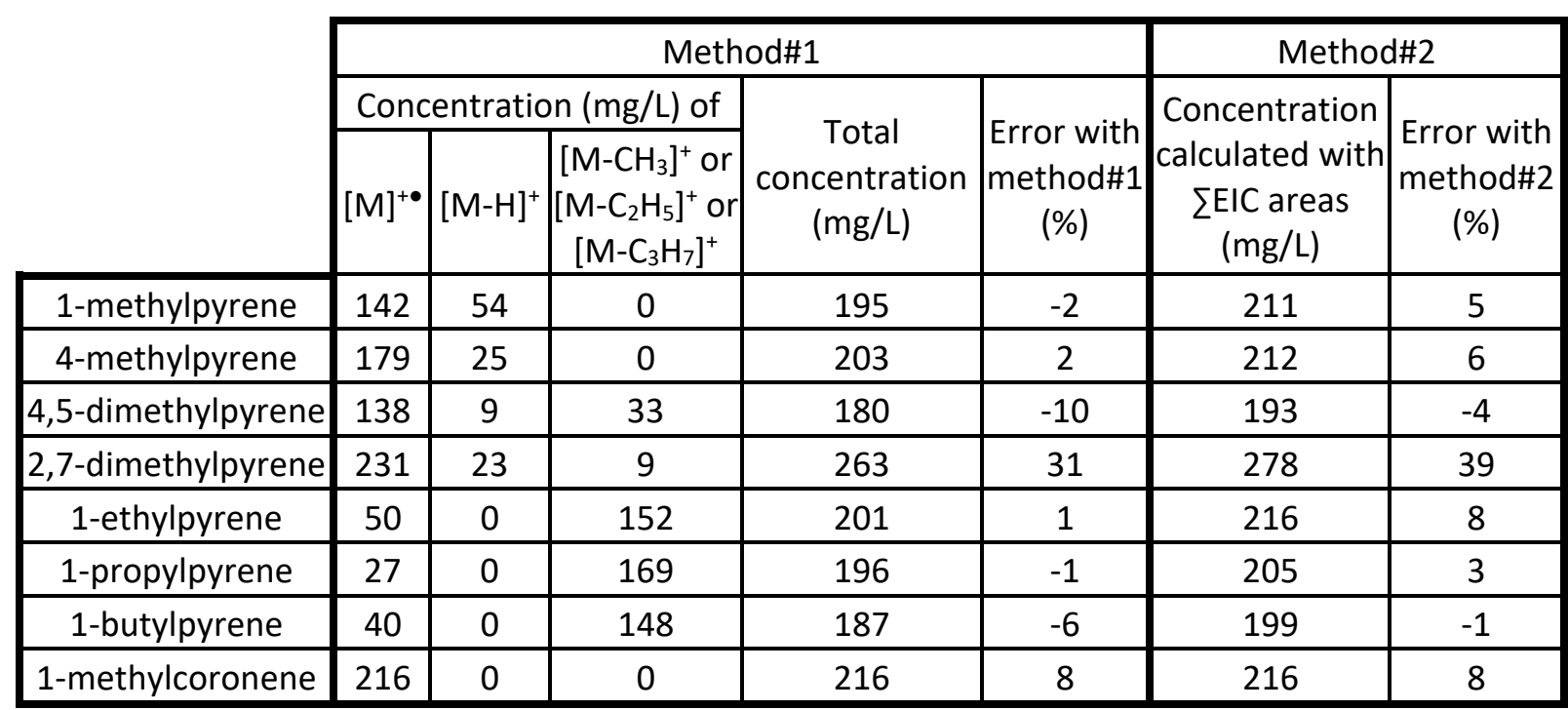

685

Table 5: UV quantification of alkylated pyrenes

\begin{tabular}{|c|c|c|c|}
\cline { 2 - 4 } \multicolumn{1}{c|}{} & $\begin{array}{c}\text { UV } \\
\text { maximum } \\
(\mathrm{nm})\end{array}$ & $\begin{array}{c}\text { Concentration } \\
\text { calculated with UV } \\
\text { detection (mg/L) }\end{array}$ & $\begin{array}{c}\text { Error } \\
(\%)\end{array}$ \\
\hline 1-methylpyrene & 336.6 & 153 & -24 \\
\hline 4-methylpyrene & 330.7 & 157 & -22 \\
\hline 4,5-dimethylpyrene & 334.2 & 103 & -48 \\
\hline 2,7-dimethylpyrene & 331.9 & 168 & -16 \\
\hline 1-ethylpyrene & 335.4 & 147 & -27 \\
\hline 1-propylpyrene & 336.6 & 140 & -30 \\
\hline 1-butylpyrene & 336.6 & 138 & -31 \\
\hline
\end{tabular}

687

Table 6: Contents $(\mathrm{mg} / \mathrm{kg}$ ) of PAHs $\pm 30 \%$ in six VGO samples according to their DBE (ND: nondetected)

\begin{tabular}{|c|c|c|c|c|c|c|}
\hline DBE & $\begin{array}{c}\text { VGO 1 } \\
\text { SRVGO }\end{array}$ & $\begin{array}{c}\text { VGO 2 } \\
\text { SRVGO }\end{array}$ & $\begin{array}{c}\text { VGO 3 } \\
\text { SRVGO }\end{array}$ & $\begin{array}{c}\text { VGO 4 } \\
\text { SRVGO }\end{array}$ & $\begin{array}{c}\text { VGO 5 } \\
\text { DCVGO }\end{array}$ & $\begin{array}{c}\text { VGO 6 } \\
\text { Blend }\end{array}$ \\
\hline $\mathbf{1 2}$ & 14357 & 10090 & 6296 & 8240 & 11905 & 13315 \\
\hline $\mathbf{1 3}$ & 6215 & 4732 & 2382 & 4521 & 8748 & 7517 \\
\hline $\mathbf{1 4}$ & 2909 & 1808 & 800 & 2098 & 6753 & 3418 \\
\hline $\mathbf{1 5}$ & 952 & 1085 & 980 & 1749 & 3022 & 1616 \\
\hline $\mathbf{1 6}$ & 13 & 85 & 91 & 234 & 1933 & 501 \\
\hline $\mathbf{1 7}$ & 107 & 95 & 89 & 135 & 1916 & 767 \\
\hline
\end{tabular}




\begin{tabular}{|c|c|c|c|c|c|c|}
\hline $\mathbf{1 8}$ & ND & $<10$ & $<10$ & 34 & 614 & 54 \\
\hline $\mathbf{1 9}$ & ND & ND & ND & ND & 65 & 24 \\
\hline $\mathbf{2 0}$ & ND & ND & ND & ND & 41 & $<10$ \\
\hline Total & 24554 & 17902 & 10642 & 17010 & 34998 & 27214 \\
\hline
\end{tabular}

690 